\title{
PULMONARY EMBOLISM
}

\author{
Major D. E. BRADFORD, \\ M.B., Ch.B., M.R.C.P.(Edin.), D.T.M.\& H., R.A.M.C. \\ Military Wing, Musgrave Park Hospital, Belfast
}

FoLLOWING the introduction of oral contraceptives there has been an increasing number of reports of intravascular thrombosis occurring in women taking these drugs. The entire problem is reviewed by Nevin et al (1965) who describe a further three patients. Most of the case reports have been concerned with the development of thrombophlebitis and pulmonary embolism, but one of the difficulties in establishing a relationship between any drug and this clinical presentation is that pulmonary embolism does occur in previously fit individuals (Cohen and Daly 1957). A fit active service population could be expected to provide a small number of such cases and examination of available case notes at the Q ueen Alexandra Military Hospital from January 1960 to June 1962 revealed three male patients with pulmonary embolism unrelated to surgical operation, heart disease, neoplasm or superficial thrombophlebitis.

From 1962 to the present no further male patients have been seen but a 34 year old female warrant officer has been treated for deep vein thrombosis and extensive pulmonary embolism "occurring while taking Enavid (norethynodrel and mestranol) for endometriosis (Bradford"1965). Because of the increasing interest in this condition the three cases are reported here in detail:

\section{Case Reports}

Case No. 1

An officer aged 25 years was admitted to hospital on 3rd December 1960 with a 24 hours history of right sided pleuritic pain and dyspnoea on exertion. He described an influenza like illness two weeks before admission and his only previous hospital admissions were for jaundice and diarrhoea 4 years before.

Initial examination showed him to be afebrile with decreased movement of the right lower chest. There was impaired percussion note and diminished breath sounds over the right lung base. The cardiovascular system was normal and his blood pressure was $130 / 80 \mathrm{~mm} \mathrm{Hg}$. There was at this time no evidence of deep or superficial vein thrombosis in his legs.

The following investigations were made; haemoglobin $12.4 \mathrm{G} \%(85 \%)$, total white cell count $9,000 / \mathrm{c} . \mathrm{cm}$ with a normal differential count, erythrocyte sedimentation rate $16 \mathrm{~mm} / \mathrm{hr}$. (Westergren), -Heaf test negative. Chest X-ray showed only a slight increase in right basal markings, and sputum culture subsequently failed to grow pathogens.

$\mathrm{He}$ was treated for right basal pneumonia with crystalline penicillin 500,000 units 6 hourly but in spite of therapy his chest pain remained. Seven days after admission he developed a right sided pleural effusion and 24 hours later a generalised erythema accompanied by fever. His total white cell count increased to $17,450 / \mathrm{c}$.cm with $15 \%$ eosinophils. Both blood examination for L.E. cells and stool examination for parasites were negative. The erythema was considered to be due to a penicillin sensitivity and the antibiotic was stopped.

He remained under observation with minimal improvement until on the evening of 18 th December he suddenly became dyspnoeic and cyanosed with sweating and right lower chest pain. Tachycardia of 120 per minute with a blood pressure of $100 / 60 \mathrm{~mm} \mathrm{Hg}$. was noted. The heart sounds were normal and there were no murmurs. There was impaired air'entry and dullness at his right lung base. Chest $X$-ray showed an opacity at the right base with a small pleural effusion. On the electrocardiogram there were deep $S$ waves in Lead 1 and $V^{1}$ to $V^{2}$, with sharply inverted $T$ waves in Lead $3, V F$ and $V^{1}$ suggestive of pulmonary embolism. Repeated examinations of his legs at this time failed to show any abnormality.

Anticoagulant therapy was started with gradual improvement in both clinical and radiological condition. On 17 th February 1961 his chest X-ray was normal. Therapy was stopped on 27 th February 1961 and the patient discharged from hospital. 
Case No. 2

An officer aged 37 years was first seen on 6th February 1956 with three days history of right sided pleuritic pain. On the day of admission to hospital he produced a small amount of blood streaked sputum. Apart from influenza two weeks before admission his previous health had been good.

Examination found him febrile, temperature of $100^{\circ} \mathrm{F}$, with dullness and impaired air entry at the right lung base. His cardiovascular system was normal, with a blood pressure of $130 / 90 \mathrm{~mm} \mathrm{Hg}$. and there was no evidence of venous disease in his legs.

Investigations included: total white cell count $8,000 / \mathrm{c.cm}$. with a normal differential count; erythrocyte sedimentation rate of $45 \mathrm{~mm} / \mathrm{hr}$. (Westergren). Chest X-ray showed an opacity at the right base with the left base obscured by a small effusion. Aspiration of the left pleural cavity produced a few ml. of blood stained fluid which was sterile on culture.

The patient was treated with penicillin with no change in his chest signs or in his fever. Five days after admission he complained of tenderness in his right leg and on examination was found to have a thrombosis of the long saphenous vein. He was given anticoagulant therapy from the 11th February to 27th February 1956 when a further chest X-ray showed clearing of the right lung base. He was discharged from hospital only to be readmitted on 12th March 1956 with extension of his right long saphenous vein thrombosis. Ligation of this vessel was carried out on 16 th March and was followed two days later by left sided chest pain with clinical and X-ray signs of left basal consolidation. In spite of his past history this was regarded as a pneumonia, until 25th March when he developed swelling of his right calf with tenderness and a positive Homans' test. Anticoagulant treatment was given, on this occasion, for a total of six weeks.

In November 1957 he was again in hospital with a deep vein thrombosis in his left calf and received anticoagulants for 2 weeks. January 1958 saw him admitted yet again, this time with a right sided pulmonary embolism. Anticoagulants were given, he was discharged from hospital in April 1958, and treatment was continued as an out-patient until January 1960. At follow up he was found to be in robust health with no residual involvement of chest or legs.

Case. No. 3

A sergeant aged 33 years entered hospital on 11th January 1960 with a history of right sided chest and shoulder pain of two days duration. There was no haemoptysis or dyspneoa. Examination found him febrile, temperature $101^{\circ} \mathrm{F}$, with dullness and absent breath sounds at the right lung base. The heart was normal and there was no peripheral venous thrombosis.

Investigations included a haemoglobin of $15.4 \mathrm{G} \%(104 \%)$, total white blood count $12,200 / \mathrm{c} . \mathrm{cm}$ with a normal differential count, and sedimentation rate of $1 \mathrm{~mm} / \mathrm{hr}$. Chest X-ray showed increased markings in the right lower lobe with blurring of the right costophrenic angle. The electrocardiogram was normal.

A diagnosis of right lower lobe pneumonia was made and the patient given $500 \mathrm{mgm}$ of tetracycline six hourly. Over the first six days in hospital he remained febrile and developed a right sided pleural effusion. Aspiration of his right chest provided blood stained fluid for culture which was sterile. On 16th January he developed signs of deep vein thrombosis in the right calf. Chest X-ray then showed a raised right diaphragm, a little fluid in the major fissure and a peripheral shadow in the.right lower zone. Anticoagulant treatment was now started. The patient was discharged from hospital on 4th March 1960 and continued taking anticoagulants for two years, during which time he remained perfectly well.

\section{Discussion}

Pulmonary embolism, occurring in fit ambulant individuals, has been reported from time to time (Homans 1943; Cohen and Daly 1957; Petch 1958; and Stevens 1961). It is not new to Army medicine in that Hampton et al (1945) described ten cases in American service personnel and Whyte (1961) recorded pulmonary embolism in a fit medical officer of 24 years who regularly played rugby. The very apt term " unheralded pulmonary embolism " was used by Cohen and Daly (1957) who drew attention to the lack of response to antibiotics and to the fact that the sputum, although blood stained, is not purulent. All three patients reported here had a sterile pleural effusions and all were initially treated as pneumonia.

The main source of embolus appears to be thrombosis in the deep veins of the lower leg as is shown often by the development of clinical deep vein thrombosis some time after embolism. Hampton et al. (1945) showed, by phlebography, deep vein obstruction in three of their patients despite the absence of clinical signs. The length of time before leg symptoms appear is however very variable (Stevens 1961) and occasionally, as in Case No. 1, leg symptoms or signs are not present at any time. Foster (1965) is of the opinion that the pelvic veins could provide a fruitful source of emboli and regards the presence of phleboliths as evidence of previous thrombosis in these veins. 
The question as to why the thrombosis occurs in the first place is difficult to answer. Whyte (1961) found the only common factor to be recent strenuous exercise. No record is available of the previous activity of the three patients described but an assessment of the activity prior to admission of future patients may be profitable. Two of the three patients here had an influenza like illness just prior to admission and although short periods in bed are not likely to produce venous thrombosis this may have contributed, in some way, to the final picture:

\section{Summary}

Pulmonary embolism. occurring in three previously fit and active individuals is described. All three were initially treated for pneumonia with no response to antibiotics and all three had sterile pleural effusions. The diagnosis of pulmonary embolism is one that must always be considered in those patients with acute chest complaints particularly in the absence of purulent sputum and in those instances of "pneumonia" showing no response to antibiotics.

BRADFORD, D. E. (1965). Lancet. 2, 902.

\section{REFERENCES}

CoHEN, H. and Daly, J. J. (1957). Brit. Med. J. 2, 1209.

FosTER, H. (1965). 'Lancet. 2, 790.

Hampton, A. O., Prodani, A. G. and KING, J. K. (1945). Bull. John Hopk. Hosp. 76, 245.

Homans, J. (19.43). New Eng. J. Med. 229, 309.

Nevin, N. C., Elmes, P. C. and WeAver, J. A. (1965). Brit. Med. J. 1, 1586.

PETCH, C. P. (1958). Lancet. 1, 741.

STEVENS, A. E. (1961). Lancet. 2, 1005.

WhYTE, A. G. D. (1961): Lancet. 1, 886.

\section{Order of St. John of Jerusalem}

Recent appointments to, or promotions in the Order, include:

Officers (Brothers)

Brigadier A. MacLennan, O.B.E.; Colonel N. G. G. Talbot, O.B.E., T.D.; Colonel

R. I. Mitchell, O.B.E.; Colonel J. H. Bennett.

\section{LEISHMAN CENTENARY NUMBER}

We have been asked to publish the names of those responsible for the pathology demonstrations-this Journal, (1966), 111, 18.

\section{Department of Pathology}

Demonstrations of Leishman's life and work: Colonel R. M. Vanreenan, M.B., M.R.C.P.(Edin.), M.C.Path., Professor of Pathology; Lieutenant-Colonel R. J. C. Hart, M.B., B.S., M.C.Path, Dip.Bact., Lecturer in Pathology; Lieutenant-Colonel J. E. Noble, M.R.C.S., L.R.C.P., M.C.Path., Officer Commanding The David Bruce Laboratories; Dr. A. J. Duggan, M.D., Director, The Wellcome Museum of Medical Science and Mr. R. M. Leach, M.B.E.

Demonstrations of Biochemistry: Miss B. Godsmark, B.Sc.

Demonstrations of the Army Tumour Registry and Exfoliative Cytology Service: Lieutenant-Colonel J. Gatt, M.D., M.Sc., M.R.C.P.(Edin.), M.C.Path and Major D. M. Robinson, M.D., Medical Corps, U.S. Army. 


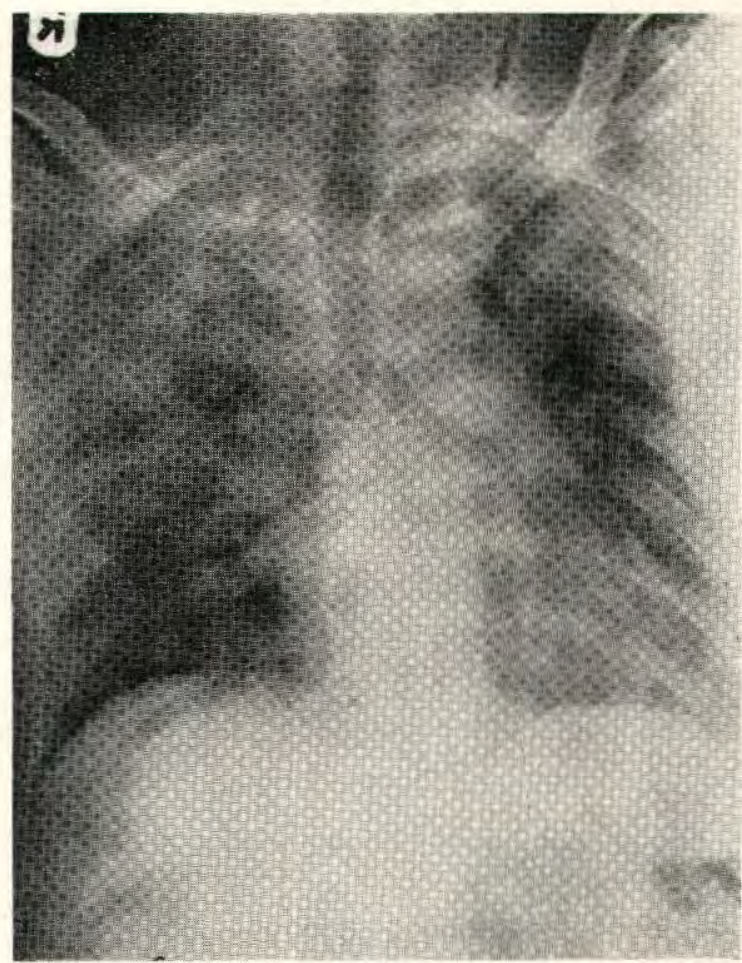

Fig. 1.

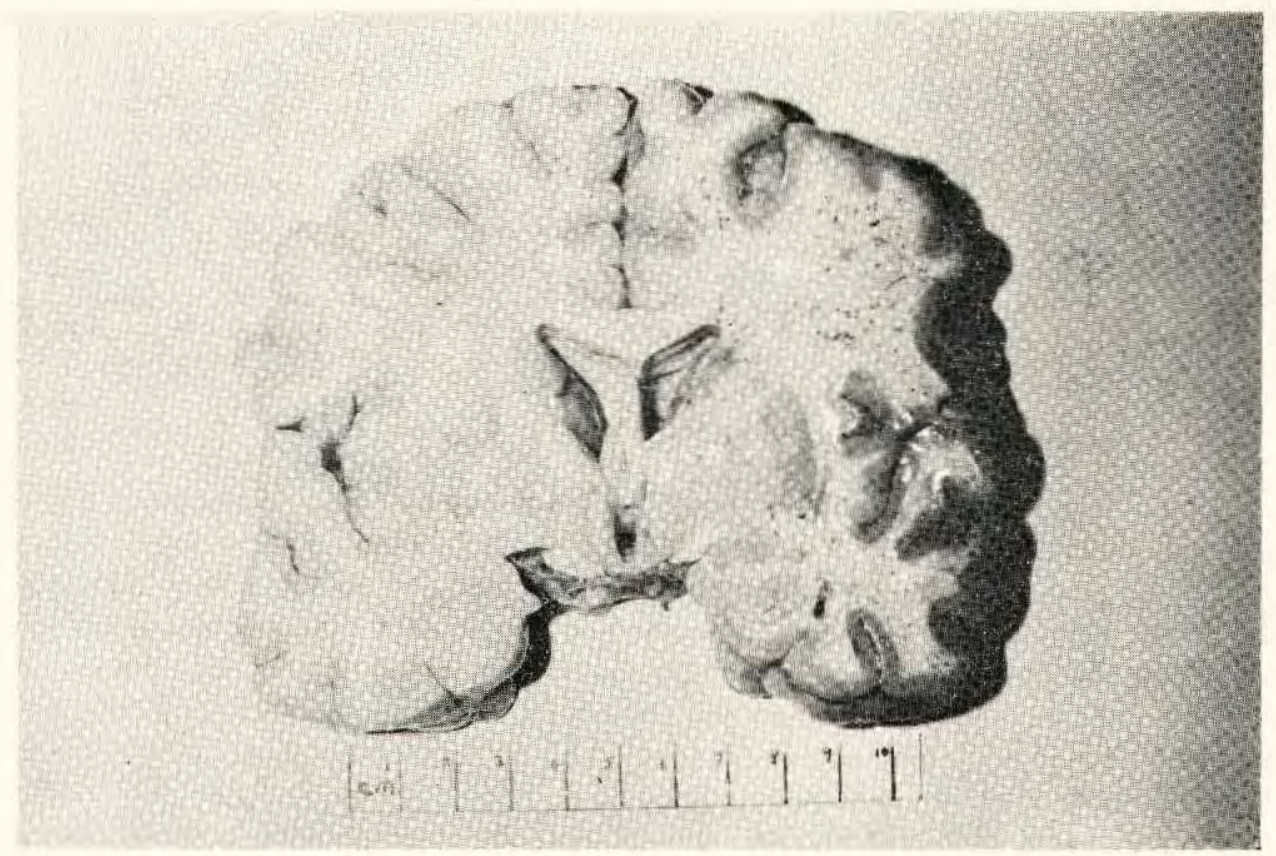

Fig. 2. Brain Coronal section showing Fat Emboli 